\title{
Suzuki Cross-Couplings of Unactivated Secondary Alkyl Bromides and Iodides
}

\author{
Jianrong (Steve) Zhou and Gregory C. Fu* \\ Department of Chemistry, \\ Massachusetts Institute of Technology, Cambridge, Massachusetts 02139 \\ Supporting Information
}

\section{General}

The following reagents were purchased: $\mathrm{Ni}(\operatorname{cod})_{2}$ (Strem), bathophenanthroline (Lancaster), KOt-Bu (Strem), s-butanol (anhydrous; Aldrich), exo-2-bromonorbornane (exo:endo 115:1; Aldrich), 1-bromobenzocyclobutane (Aldrich), isopropyl bromide (AlfaAesar), cyclopentyl bromide (Alfa-Aesar), trans-2-bromo-1-hydroxylindane (Lancaster), cyclohexyl bromide (Alfa-Aesar), cyclohexyl iodide (Lancaster), s-butyl iodide (TCI), neopentyl iodide (Aldrich), 1-iodo-3-methylbutane (Aldrich), phenylboronic acid (Frontier Scientific), $p$-tolylboronic acid (Frontier Scientific), 4-methoxyphenylboronic acid (Frontier Scientific), 4-cyanophenylboronic acid (Frontier Scientific), 4trifluoromethylphenylboronic acid (Frontier Scientific), 3-thiopheneboronic acid (Aldrich), $\mathrm{N}$-methylindole-5-boronic acid (Frontier Scientific), 3-chlorophenylboronic acid (Frontier Scientific), 3,4-methylenedioxyphenylboronic acid (Frontier Scientific), trans- $\beta$-styreneboronic acid (Frontier Scientific), 4-methylthiophenylboronic acid (Frontier Scientific), (E)-1-octen-1-ylboronic acid (Aldrich). endo-2-Bromonorbornane [13237-87-1] (endo:exo 32:1) was prepared according to a literature procedure. ${ }^{1}$ The liquid alkyl halides were purged with argon prior to use. Other reagents were used as received.

All reactions were conducted in oven-dried glassware under an inert atmosphere with magnetic stirring. 


\section{Preparation of Substrates}

trans-2-Bromo-1-( $t$-butyldimethylsiloxyl)indane. Imidazole $(0.96 \mathrm{~g}, 14 \mathrm{mmol})$ was added to a solution of trans-2-bromo-1-hydroxylindane $(2.00 \mathrm{~g}, 9.4 \mathrm{mmol})$ and TBSCl $(1.83 \mathrm{~g}, 12.2 \mathrm{mmol})$ in $\mathrm{CH}_{2} \mathrm{Cl}_{2}(50 \mathrm{~mL})$ at r.t. This reaction mixture was stirred overnight, and then the resulting yellow suspension was filtered through a pad of silica gel (washed with 10:1 hexane/EtOAc). The filtrate was concentrated, and the residue was purified by flash chromatography (hexane), which provided $2.72 \mathrm{~g}(88 \%)$ of the desired product as a yellow oil.

${ }^{1} \mathrm{H}$ NMR $\left(400 \mathrm{MHz}, \mathrm{CDCl}_{3}\right): 7.33-7.22(\mathrm{~m}, 4 \mathrm{H}), 5.33(\mathrm{~d}, J=5.7 \mathrm{~Hz}, 1 \mathrm{H}), 4.27(\mathrm{dd}, J=$ $13.8,7.4 \mathrm{~Hz}, 1 \mathrm{H}), 3.58(\mathrm{dd}, J=16.1,7.4 \mathrm{~Hz}, 1 \mathrm{H}), 3.22(\mathrm{dd}, J=16.1,7.5 \mathrm{~Hz}, 1 \mathrm{H}), 0.99$ (s, $9 \mathrm{H}), 0.29(\mathrm{~s}, 3 \mathrm{H}), 0.26(\mathrm{~s}, 3 \mathrm{H})$.

${ }^{13} \mathrm{C} \mathrm{NMR}\left(400 \mathrm{MHz}, \mathrm{CDCl}_{3}\right): 143.2,139.7,128.6,127.5,124.6,124.3,84.0,55.0,40.9$ ， $26.1,18.4,-3.6,-4.1$.

IR (thin film): 2956, 2929, 2894, 2857, 1471, 1462, 1362, $1257 \mathrm{~cm}^{-1}$.

HRMS (EI): Calcd for $\mathrm{C}_{15} \mathrm{H}_{22} \mathrm{BrSiO}(\mathrm{M}-\mathrm{H})^{+}:$325.0623. Found: 325.0628 . 


\section{Suzuki Cross-Coupling Reactions (Tables 2 and 3)}

Procedure: no glove box. $\mathrm{Ni}(\mathrm{cod})_{2}(5.6 \mathrm{mg}, 0.020 \mathrm{mmol})$, bathophenanthroline (13.2 $\mathrm{mg}, 0.040 \mathrm{mmol})$, the arylboronic acid $(0.60 \mathrm{mmol})$, and $\mathrm{KO} t-\mathrm{Bu}(90 \mathrm{mg}, 0.80 \mathrm{mmol})$ were added to a vial equipped with a stir bar. The vial was evacuated/refilled with argon three times, and then $s$-butanol $(3.0 \mathrm{~mL})$ was added. The mixture was stirred at r.t. for $10 \mathrm{~min}$, and to the resulting deep-purple solution was added the alkyl halide $(0.50 \mathrm{mmol})$. The reaction mixture was stirred at $60^{\circ} \mathrm{C}$ for $5 \mathrm{~h}$, and then it was passed through a short pad of silica gel (to remove $s$-butanol and polar compounds). The filtrate was concentrated, and the residue was purified by flash chromatography.

Procedure: with a glove box. In a glove box, $\mathrm{Ni}(\mathrm{cod})_{2}(5.6 \mathrm{mg}, 0.020 \mathrm{mmol})$, bathophenanthroline $(13.2 \mathrm{mg}, 0.040 \mathrm{mmol})$, the arylboronic acid $(0.60 \mathrm{mmol}), \mathrm{KOt}-\mathrm{Bu}$ (90 mg, $0.80 \mathrm{mmol})$, and s-butanol $(3.0 \mathrm{~mL})$ were added to a vial equipped with a stir bar. The mixture was stirred at r.t. for $10 \mathrm{~min}$, and to the resulting deep-purple solution was added the alkyl halide $(0.50 \mathrm{mmol})$. The vial was removed from the glove box, and the reaction mixture was stirred at $60^{\circ} \mathrm{C}$ for $5 \mathrm{~h}$. Then, it was passed through a short pad of silica gel (to remove s-butanol and polar compounds), and the filtrate was concentrated. The residue was purified by flash chromatography.

exo-2-Phenylnorbornane [52752-81-5] (Table 2, entry 1). exo-2-Bromonorbornane (88 mg, $0.50 \mathrm{mmol}$; exo:endo 115:1) and phenylboronic acid (74 mg, $0.60 \mathrm{mmol}$ ) were used. Solvent for chromatography: hexane. Colorless oil (no glove box: $64 \mathrm{mg}, 74 \%$; glove box: $63 \mathrm{mg}, 73 \%)$. ${ }^{1} \mathrm{H}$ NMR revealed a product exo:endo ratio of $>50: 1$. The assignment of product stereochemistry is based on comparison with reported ${ }^{1} \mathrm{H}$ and ${ }^{13} \mathrm{C}$ NMR spectra. ${ }^{2}$

${ }^{1} \mathrm{H}$ NMR $\left(400 \mathrm{MHz}, \mathrm{CDCl}_{3}\right):$ 7.34-7.31 $(\mathrm{m}, 2 \mathrm{H}), 7.28-7.26(\mathrm{~m}, 2 \mathrm{H}), 7.21-7.18(\mathrm{~m}, 1 \mathrm{H})$, $2.79(\mathrm{dd}, J=8.7,5.8 \mathrm{~Hz}, 1 \mathrm{H}), 2.40(\mathrm{~d}, J=3.1 \mathrm{~Hz}, 2 \mathrm{H}), 1.85-1.79(\mathrm{~m}, 1 \mathrm{H}), 1.74-1.68(\mathrm{~m}, 1 \mathrm{H})$, $1.65-1.57(\mathrm{~m}, 3 \mathrm{H}), 1.43-1.30(\mathrm{~m}, 2 \mathrm{H}), 1.23(\mathrm{dd}, J=8.2 \mathrm{~Hz}, 1.5 \mathrm{~Hz}, 1 \mathrm{H})$.

${ }^{13} \mathrm{C}$ NMR $\left(400 \mathrm{MHz}, \mathrm{CDCl}_{3}\right)$ : 147.8, 128.4, 127.3, 125.6, 47.5, 43.1, 39.3, 37.0, 36.3, 30.8, 29.1.

exo-2-Phenylnorbornane [52752-81-5] (Table 2, entry 2). endo-2-Bromonorbornane (88 mg, $0.50 \mathrm{mmol}$; endo:exo 32:1) and phenylboronic acid (74 mg, $0.60 \mathrm{mmol}$ ) were used. Solvent for chromatography: hexane. Colorless oil (no glove box: $62 \mathrm{mg}, 71 \%$; glove box: $64 \mathrm{mg}, 74 \%$ ). ${ }^{1} \mathrm{H}$ NMR revealed a product exo:endo ratio of $>50: 1$. The assignment of product stereochemistry is based on comparison with reported ${ }^{1} \mathrm{H}$ and ${ }^{13} \mathrm{C}$ NMR spectra. ${ }^{2}$

1-p-Tolylbenzocyclobutene (Table 2, entry 3). 1-Bromobenzocyclobutane (92 mg, $0.50 \mathrm{mmol})$ and $p$-tolylboronic acid $(81 \mathrm{mg}, 0.60 \mathrm{mmol})$ were used. Solvent for chromatography: hexane. Colorless oil (no glove box: $87 \mathrm{mg}, 90 \%$; glove box: $82 \mathrm{mg}$, $84 \%)$.

${ }^{1} \mathrm{H}$ NMR $\left(400 \mathrm{MHz}, \mathrm{CDCl}_{3}\right): 7.36-7.31(\mathrm{~m}, 2 \mathrm{H}), 7.24-7.17(\mathrm{~m}, 6 \mathrm{H}), 4.73(\mathrm{dd}, J=5.5,2.6$ $\mathrm{Hz}, 1 \mathrm{H}), 3.77(\mathrm{dd}, J=13.9,5.6 \mathrm{~Hz}, 1 \mathrm{H}), 3.13(\mathrm{dd}, J=13.9,2.6 \mathrm{~Hz}, 1 \mathrm{H}), 2.40(\mathrm{~s}, 3 \mathrm{H})$.

${ }^{13} \mathrm{C}$ NMR $\left(400 \mathrm{MHz}, \mathrm{CDCl}_{3}\right): 148.1,144.4,140.0,136.1,129.3,127.7,127.3,127.0$, 123.4, 122.9, 47.4, 40.3, 21.3.

IR (thin film): 3021, 2921, 1661, 1513, $1459 \mathrm{~cm}^{-1}$.

HRMS (EI): Calcd for $\mathrm{C}_{15} \mathrm{H}_{14}\left(\mathrm{M}^{+}\right)$: 194.1090. Found: 194.1090. 
4-Isopropylanisole [4132-48-3] (Table 2, entry 4). Isopropyl bromide (62 mg, 0.50 $\mathrm{mmol}$ ) and 4-methoxyphenylboronic acid $(99 \mathrm{mg}, 0.60 \mathrm{mmol})$ were used. Solvent for chromatography: hexane/ethyl ether 100:1. Colorless oil (no glove box: $51 \mathrm{mg}, 68 \%$; glove box: $50 \mathrm{mg}, 67 \%)$.

${ }^{1} \mathrm{H} \mathrm{NMR}\left(400 \mathrm{MHz}, \mathrm{CDCl}_{3}\right)$ : 7.25-7.21 (m, 2H), 6.94-6.90 (m, 2H), $3.87(\mathrm{~s}, 3 \mathrm{H}), 2.94$ (heptet, $J=6.9 \mathrm{~Hz}, 1 \mathrm{H}), 1.30(\mathrm{~d}, J=6.9 \mathrm{~Hz}, 6 \mathrm{H})$.

${ }^{13} \mathrm{C}$ NMR $\left(400 \mathrm{MHz}, \mathrm{CDCl}_{3}\right): 157.8,141.3,127.5,113.9,55.5,33.5,24.4$.

3-Cyclopentylbenzonitrile (Table 2, entry 5). Cyclopentyl bromide (76 mg, 0.50 $\mathrm{mmol})$ and 3-cyanophenylboronic acid $(89 \mathrm{mg}, 0.60 \mathrm{mmol})$ were used. Solvent for chromatography: hexane/ethyl ether 100:1. Colorless oil (no glove box: $38 \mathrm{mg}$, 44\%; glove box: $40 \mathrm{mg}, 47 \%)$.

${ }^{1} \mathrm{H} \mathrm{NMR}\left(400 \mathrm{MHz}, \mathrm{CDCl}_{3}\right): 7.54(\mathrm{~s}, 1 \mathrm{H}), 7.50-7.48(\mathrm{~m}, 2 \mathrm{H}), 7.41-7.37(\mathrm{~m}, 1 \mathrm{H}), 3.03$ $(\mathrm{m}, 1 \mathrm{H}), 2.15-2.08(\mathrm{~m}, 2 \mathrm{H}), 1.88-1.80(\mathrm{~m}, 2 \mathrm{H}), 1.78-1.69(\mathrm{~m}, 2 \mathrm{H}), 1.63-1.53(\mathrm{~m}, 2 \mathrm{H})$.

${ }^{13} \mathrm{C} \mathrm{NMR}\left(400 \mathrm{MHz}, \mathrm{CDCl}_{3}\right): 148.1,132.0,130.9,129.6,129.2,119.4,112.3,45.6,34.6$, 25.6 .

IR (thin film): 2955, 2869, 2228, $1483 \mathrm{~cm}^{-1}$.

HRMS (EI): Calcd for $\mathrm{C}_{12} \mathrm{H}_{13} \mathrm{~N}\left(\mathrm{M}^{+}\right)$: 171.1043 . Found: 171.1035.

trans-1-t-Butyldimethylsiloxy-2-(4-trifluoromethylphenyl)indane (Table 2, entry 6). trans-2-Bromo1-t-butyldimethylsiloxylindane $(164 \mathrm{mg}, 0.50 \mathrm{mmol})$ and 4 trifluoromethylphenylboronic acid $(114 \mathrm{mg}, 0.60 \mathrm{mmol})$ were used. Solvent for chromatography: hexane/ethyl ether 100:1. Colorless oil (no glove box: $123 \mathrm{mg}, 63 \%$; glove box: $118 \mathrm{mg}, 60 \%)$. ${ }^{1} \mathrm{H}$ NMR revealed a product trans:cis ratio of $>50: 1.3$

${ }^{1} \mathrm{H}$ NMR $\left(400 \mathrm{MHz}, \mathrm{CDCl}_{3}\right): 7.62(\mathrm{~d}, J=8.1 \mathrm{~Hz}, 2 \mathrm{H}), 7.47(\mathrm{~d}, J=8.1 \mathrm{~Hz}, 2 \mathrm{H}), 7.33-7.25$ $(\mathrm{m}, 4 \mathrm{H}), 5.29(\mathrm{~d}, J=7.7 \mathrm{~Hz}, 1 \mathrm{H}), 3.50(\mathrm{dd}, J=17.8,8.2 \mathrm{~Hz}, 1 \mathrm{H}), 3.36(\mathrm{dd}, J=15.7,8.2 \mathrm{~Hz}$, $1 \mathrm{H}), 3.08(\mathrm{dd}, J=15.6,9.9 \mathrm{~Hz}, 1 \mathrm{H}), 0.89(\mathrm{~s}, 9 \mathrm{H}), 0.04(\mathrm{~s}, 3 \mathrm{H}),-0.35(\mathrm{~s}, 3 \mathrm{H})$.

${ }_{13} \mathrm{C} \mathrm{NMR}\left(400 \mathrm{MHz}, \mathrm{CDCl}_{3}\right)$ : 147.2, 144.7, 140.5, 129.1, 128.7, 128.3, 127.3, 125.61, $125.57,124.8,124.1,83.9,57.3,38.3,25.9,18.2,-4.3,-4.7$.

${ }^{19} \mathrm{~F}$ NMR $\left(400 \mathrm{MHz}, \mathrm{CDCl}_{3}\right):-62.6$.

IR (thin film): 2955, 2930, 2858, 1620, $1326 \mathrm{~cm}^{-1}$.

HRMS (EI): Calcd for $\mathrm{C}_{18} \mathrm{H}_{18} \mathrm{SiOF}_{3}\left(\mathrm{M}-\mathrm{C}_{4} \mathrm{H}_{9}\right)^{+}: 335.1074$. Found: 335.1075 .

3-Cyclohexylthiophene [120659-34-9] (Table 2, entry 7). Cyclohexyl bromide (82 $\mathrm{mg}, 0.50 \mathrm{mmol}$ ) and 3-thiopheneboronic acid $(77 \mathrm{mg}, 0.60 \mathrm{mmol})$ were used. Solvent for chromatography: hexane. Colorless oil (no glove box: $52 \mathrm{mg}$, 63\%; glove box: 51 $\mathrm{mg}, 61 \%)$.

${ }^{1} \mathrm{H} \mathrm{NMR}\left(400 \mathrm{MHz}, \mathrm{CDCl}_{3}\right): 7.27(\mathrm{dd}, J=5.7,3.7 \mathrm{~Hz}, 1 \mathrm{H}), 7.02(\mathrm{~d}, J=4.9 \mathrm{~Hz}, 1 \mathrm{H}), 6.96$ $(\mathrm{d}, J=2.1 \mathrm{~Hz}, 1 \mathrm{H}), 2.67-2.62(\mathrm{~m}, 1 \mathrm{H}), 2.05-2.00(\mathrm{~m}, 2 \mathrm{H}), 1.86-1.84(\mathrm{~m}, 2 \mathrm{H}), 1.81-1.74(\mathrm{~m}$, $1 \mathrm{H}), 1.47-1.35(\mathrm{~m}, 4 \mathrm{H}), 1.32-1.24(\mathrm{~m}, 1 \mathrm{H})$.

${ }_{13} \mathrm{C} \mathrm{NMR}\left(400 \mathrm{MHz}, \mathrm{CDCl}_{3}\right): 149.3,127.2,125.1,118.5,39.7,34.4,26.8,26.4$.

$\mathrm{N}$-Methyl-5-cyclohexylindole (Table 2, entry 8). Cyclohexyl bromide $(82 \mathrm{mg}, 0.50$ $\mathrm{mmol}$ ) and $\mathrm{N}$-methylindole-5-boronic acid $(105 \mathrm{mg}, 0.60 \mathrm{mmol})$ were used. Solvent for chromatography: hexane/ethyl ether 100:1. Yellow solid (no glove box: $71 \mathrm{mg}, 67 \%$; glove box: $69 \mathrm{mg}, 65 \%)$.

${ }^{1} \mathrm{H}$ NMR $\left(400 \mathrm{MHz}, \mathrm{CDCl}_{3}\right): 7.51(\mathrm{t}, J=0.8 \mathrm{~Hz}, 1 \mathrm{H}), 7.29(\mathrm{~d}, J=11.3 \mathrm{~Hz}, 1 \mathrm{H}), 7.16(\mathrm{dd}$, $J=8.4,1.6 \mathrm{~Hz}, 1 \mathrm{H}), 7.06(\mathrm{~d}, J=3.1 \mathrm{~Hz}, 1 \mathrm{H}), 6.48(\mathrm{~d}, J=3.1 \mathrm{~Hz}, 1 \mathrm{H}), 3.81(\mathrm{~s}, 3 \mathrm{H}), 2.68-2.61$ $(\mathrm{m}, 1 \mathrm{H}), 1.98(\mathrm{~d}, J=11.9 \mathrm{~Hz}, 2 \mathrm{H}), 1.91(\mathrm{~d}, J=12.2 \mathrm{~Hz}, 2 \mathrm{H}), 1.84-1.80(\mathrm{~m}, 1 \mathrm{H}), 1.60-1.42$ $(\mathrm{m}, 4 \mathrm{H}), 1.39-1.32(\mathrm{~m}, 1 \mathrm{H})$. 
${ }^{13} \mathrm{C} \mathrm{NMR}\left(400 \mathrm{MHz}, \mathrm{CDCl}_{3}\right): 139.5,135.6,129.0,128.7,121.4,118.4,109.1,100.7,44.9$, $35.4,33.0,27.4,26.5$.

IR (KBr pellet): 2921, 2849, 1511, 1490, 1446, $1424 \mathrm{~cm}^{-1}$.

HRMS (EI): Calcd for $\mathrm{C}_{15} \mathrm{H}_{19} \mathrm{~N}\left(\mathrm{M}^{+}\right)$: 213.1512. Found: 213.1509 .

3-Cyclohexyl-1-chlorobenzene [27163-66-2] (Table 2, entry 9). Cyclohexyl bromide ( $82 \mathrm{mg}, 0.50 \mathrm{mmol}$ ) and 3-chlorophenylboronic acid $(94 \mathrm{mg}, 0.60 \mathrm{mmol}$ ) were used. Solvent for chromatography: hexane. Colorless oil (no glove box: $71 \mathrm{mg}, 75 \%$; glove box: $68 \mathrm{mg}, 70 \%)$.

${ }^{1} \mathrm{H}$ NMR $\left(400 \mathrm{MHz}, \mathrm{CDCl}_{3}\right): 7.26-7.22(\mathrm{~m}, 2 \mathrm{H}), 7.19-7.16(\mathrm{~m}, 1 \mathrm{H}), 7.12-7.10(\mathrm{~m}, 1 \mathrm{H})$, 2.54-2.48 (m, $1 \mathrm{H}), 1.89-1.86(\mathrm{~m}, 4 \mathrm{H}), 1.80-1.76(\mathrm{~m}, 1 \mathrm{H}), 1.47-1.35(\mathrm{~m}, 4 \mathrm{H}), 1.31-1.25(\mathrm{~m}$, $1 \mathrm{H})$. 26.2 .

${ }^{13} \mathrm{C}$ NMR (400 MHz, $\left.\mathrm{CDCl}_{3}\right)$ : 150.3, 134.2, 129.7, 127.2, 126.1, 125.3, 44.5, 34.5, 27.0,

Formaldedehyde 4-cyclohexyl-1,2-catechol acetal (Table 3, entry 1). Cyclohexyl iodide $(106 \mathrm{mg}, 0.50 \mathrm{mmol})$ and 3,4-methylenedioxyphenylboronic acid (100 $\mathrm{mg}, 0.60$ mmol) were used. Solvent for chromatography: hexane/ethyl ether 100:1. Colorless oil (no glove box: $63 \mathrm{mg}$, 62\%; glove box: $61 \mathrm{mg}, 60 \%$ ).

${ }^{1} \mathrm{H}$ NMR $\left(400 \mathrm{MHz}, \mathrm{CDCl}_{3}\right): 6.77-6.75(\mathrm{~m}, 2 \mathrm{H}), 6.68(\mathrm{dd}, J=8.0,1.6 \mathrm{~Hz}, 1 \mathrm{H}), 5.94(\mathrm{~s}$, $2 \mathrm{H}), 2.48-2.43(\mathrm{~m}, 1 \mathrm{H}), 1.87-1.85(\mathrm{~m}, 4 \mathrm{H}), 1.81-1.75(\mathrm{~m}, 1 \mathrm{H}), 1.45-1.33(\mathrm{~m}, 4 \mathrm{H}), 1.30-1.23$ $(\mathrm{m}, 1 \mathrm{H})$.

${ }^{13} \mathrm{C}$ NMR $\left(400 \mathrm{MHz}, \mathrm{CDCl}_{3}\right): 147.6,145.6,142.5,119.7,108.2,107.5,100.9,44.6,35.0$, 27.1, 26.3.

IR (thin film): 2925, 2851, 1503, 1490, 1441, $1247 \mathrm{~cm}^{-1}$.

HRMS (EI): Calcd for $\mathrm{C}_{13} \mathrm{H}_{16} \mathrm{O}_{2}\left(\mathrm{M}^{+}\right)$: 204.1145. Found: 204.1143 .

(E)-3-Methyl-1-phenyl-1-pentene [15325-63-0] (Table 3, entry 2). s-Butyl iodide (92 $\mathrm{mg}, 0.50 \mathrm{mmol})$ and trans $\beta$-styreneboronic acid $(89 \mathrm{mg}, 0.60 \mathrm{mmol})$ were used.

Solvent for chromatography: hexane. Colorless oil (no glove box: $52 \mathrm{mg}, 65 \%$; glove box: $49 \mathrm{mg}, 61 \%)$.

${ }^{1} \mathrm{H} \mathrm{NMR}\left(400 \mathrm{MHz}, \mathrm{CDCl}_{3}\right)$ : 7.39-7.37 $(\mathrm{m}, 2 \mathrm{H}), 7.33-7.29(\mathrm{~m}, 2 \mathrm{H}), 7.23-7.19(\mathrm{~m}, 1 \mathrm{H})$, $6.36(\mathrm{~d}, J=15.9 \mathrm{~Hz}, 1 \mathrm{H}), 6.12(\mathrm{dd}, J=15.9,7.9 \mathrm{~Hz}, 1 \mathrm{H}), 2.22(\mathrm{~m}, 1 \mathrm{H}), 1.43$ (quintet, $J=8.2$ $\mathrm{Hz}, 2 \mathrm{H}), 1.10(\mathrm{~d}, J=6.7 \mathrm{~Hz}, 3 \mathrm{H}), 0.93(\mathrm{t}, J=7.4 \mathrm{~Hz}, 3 \mathrm{H})$.

${ }_{13} \mathrm{C}$ NMR $\left(400 \mathrm{MHz}, \mathrm{CDCl}_{3}\right): 138.2,137.0,128.7,128.3,126.9,126.2,39.1,30.0,20.4$, 12.1 .

Methyl 4-neopentylphenyl thioether (Table 3, entry 3). Neopentyl iodide (100 mg, $0.50 \mathrm{mmol}$ ) and 4-methylthiophenylboronic acid (101 $\mathrm{mg}, 0.60 \mathrm{mmol})$ were used.

Solvent for chromatography: hexane/ethyl ether 100:3. Colorless oil (no glove box: 73 $\mathrm{mg}, 75 \%$; glove box: $68 \mathrm{mg}, 70 \%$ ).

${ }^{1} \mathrm{H} \mathrm{NMR}\left(400 \mathrm{MHz}, \mathrm{CDCl}_{3}\right): 7.21-7.19(\mathrm{~m}, 2 \mathrm{H}), 7.08-7.06(\mathrm{~m}, 2 \mathrm{H}), 2.50(\mathrm{~s}, 3 \mathrm{H}), 2.47(\mathrm{~s}$, $2 \mathrm{H}), 0.91(\mathrm{~s}, 9 \mathrm{H})$.

${ }^{13} \mathrm{C} \mathrm{NMR}\left(400 \mathrm{MHz}, \mathrm{CDCl}_{3}\right): 137.1,135.3,131.2,126.5,49.9,32.0,29.5,16.4$.

IR (thin film): 2920, 2864, 1495, 1476, $1363 \mathrm{~cm}^{-1}$.

HRMS (EI): Calcd for $\mathrm{C}_{12} \mathrm{H}_{18} \mathrm{~S}\left(\mathrm{M}^{+}\right)$: 194.1124. Found: 194.1119 .

(E)-2-Methyl-5-dodecene [112197-48-5] (Table 3, entry 4). 1-Iodo-3-methylbutane $(100 \mathrm{mg}, 0.50 \mathrm{mmol}$ ) and $(E)-1$-octen-1-ylboronic acid $(94 \mathrm{mg}, 0.60 \mathrm{mmol}$ ) were used. Solvent for chromatography: hexane. Colorless oil (no glove box: $57 \mathrm{mg}, 63 \%$; glove box: $56 \mathrm{mg}, 61 \%)$. 
${ }_{1}^{1} \mathrm{H}$ NMR $\left(400 \mathrm{MHz}, \mathrm{CDCl}_{3}\right): 5.42-5.40(\mathrm{~m}, 2 \mathrm{H}), 1.20$ (br s, 4H), 1.57 (heptet, $J=6.6$ $\mathrm{Hz}, 1 \mathrm{H}), 1.35-1.22(\mathrm{~m}, 10 \mathrm{H}), 0.92-0.89(\mathrm{~m}, 9 \mathrm{H})$.

${ }^{13} \mathrm{C} \mathrm{NMR}\left(400 \mathrm{MHz}, \mathrm{CDCl}_{3}\right): 130.7,130.4,39.1,32.9,32.0,30.7,29.9,29.1,27.7,22.9$, 22.7, 14.3. 


\section{References}

(1) (a) Tamao, K.; Yoshida, J.-i.; Takahashi, M.; Yamamoto, H.; Kakui, T.; Matsumoto, H.; Kurita, A.; Kumada, M. J. Am. Chem. Soc. 1978, 100, 290-292. (b) Tamao, K.; Yoshida, J.-i.; Murata, M.; Kumada, M. J. Am. Chem. Soc. 1980, 102, 3267-3269. (c) Uozumi, Y.; Hayashi, T. J. Am. Chem. Soc. 1991, 113, 9887-9888. (d) Uozumi, Y.; Lee, S.-Y.; Hayashi, T. Tetrahedron Lett. 1992, 33, 7185-7188.

(2) (a) exo-2-Phenylnorbornane: Brunel, J. M.; Heumann, A.; Buono, G. Angew. Chem., Int. Ed. 2000, 39, 1946-1949; Namyslo, J. C.; Kaufmann, D. E. Chem. Ber. 1997, 130, 1327-1331. (b) endo-2-Phenylnorbornane: Tateiwa, J.-i., Aoki, I.; Suama, M.; Uemura, S. Bull. Chem. Soc. Jpn. 1994, 67, 1170-1177.

(3) trans-1-t-Butyldimethylsiloxy-2-phenylindane was synthesized via hydroboration/oxidation of commercially available 2-phenylindene, followed by silylation of the secondary alcohol. The coupling patterns for the protons at $C_{2}$ and $C_{3}$ are essentially identical to those for the reaction product of Table 2, entry 6 . 


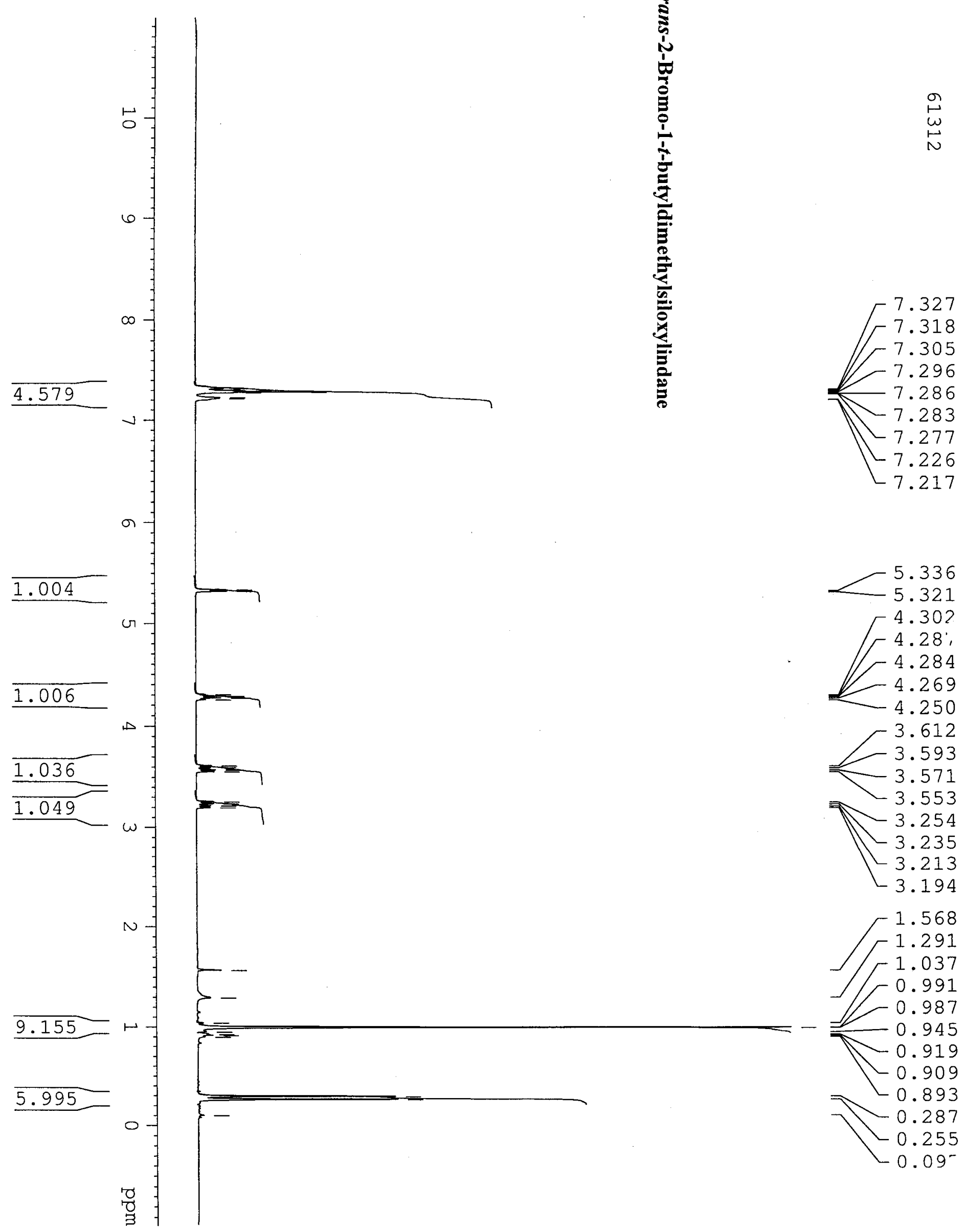




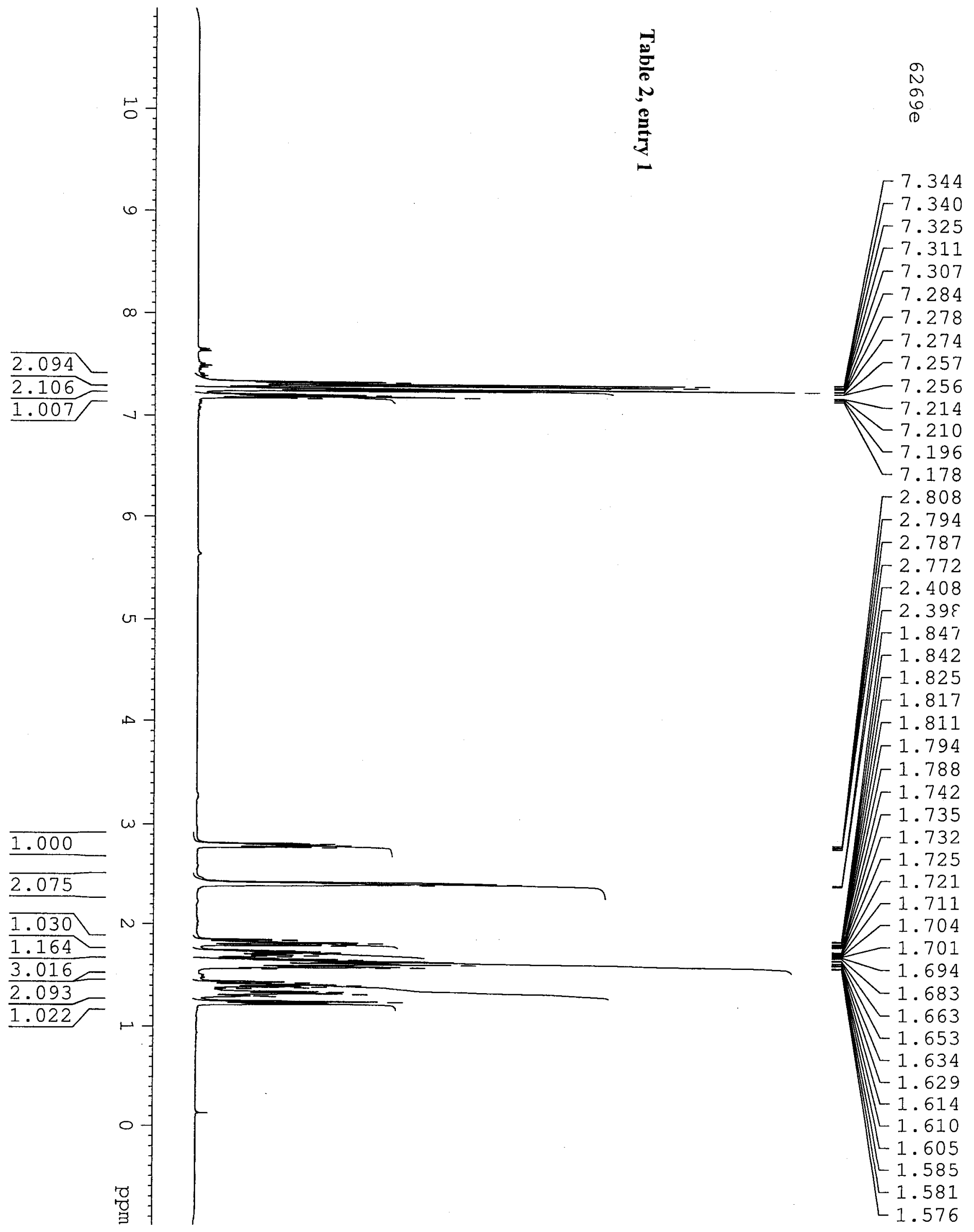




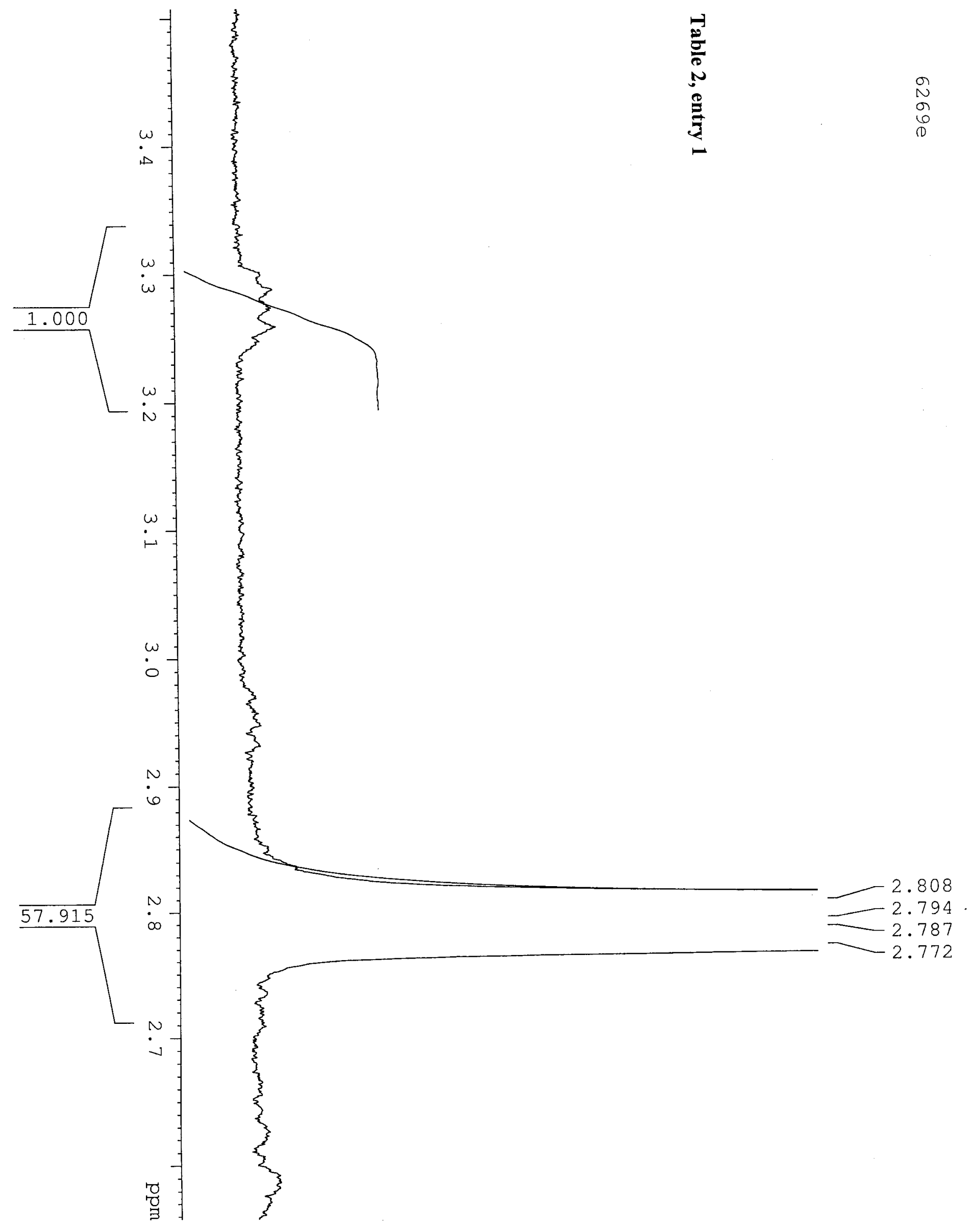




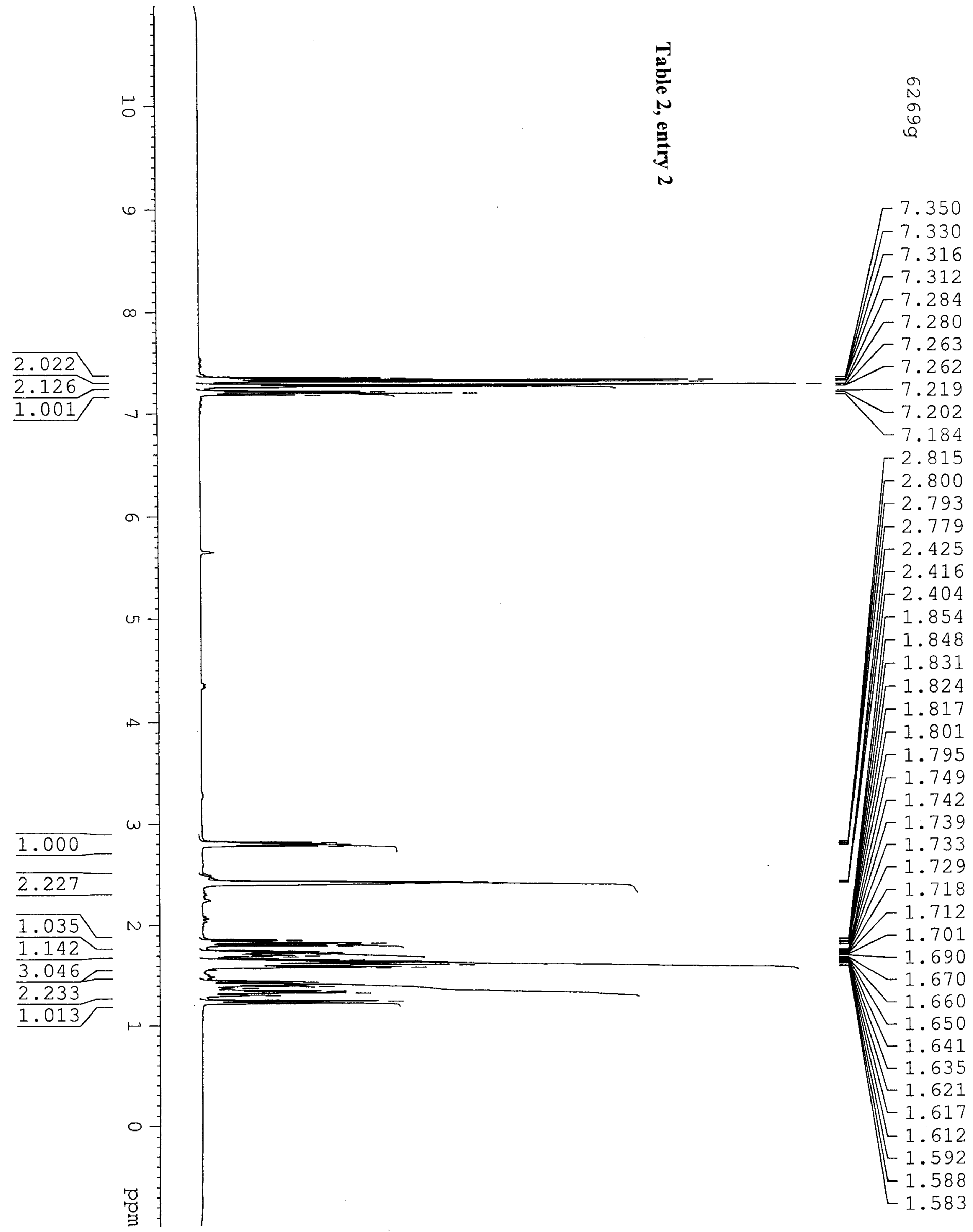




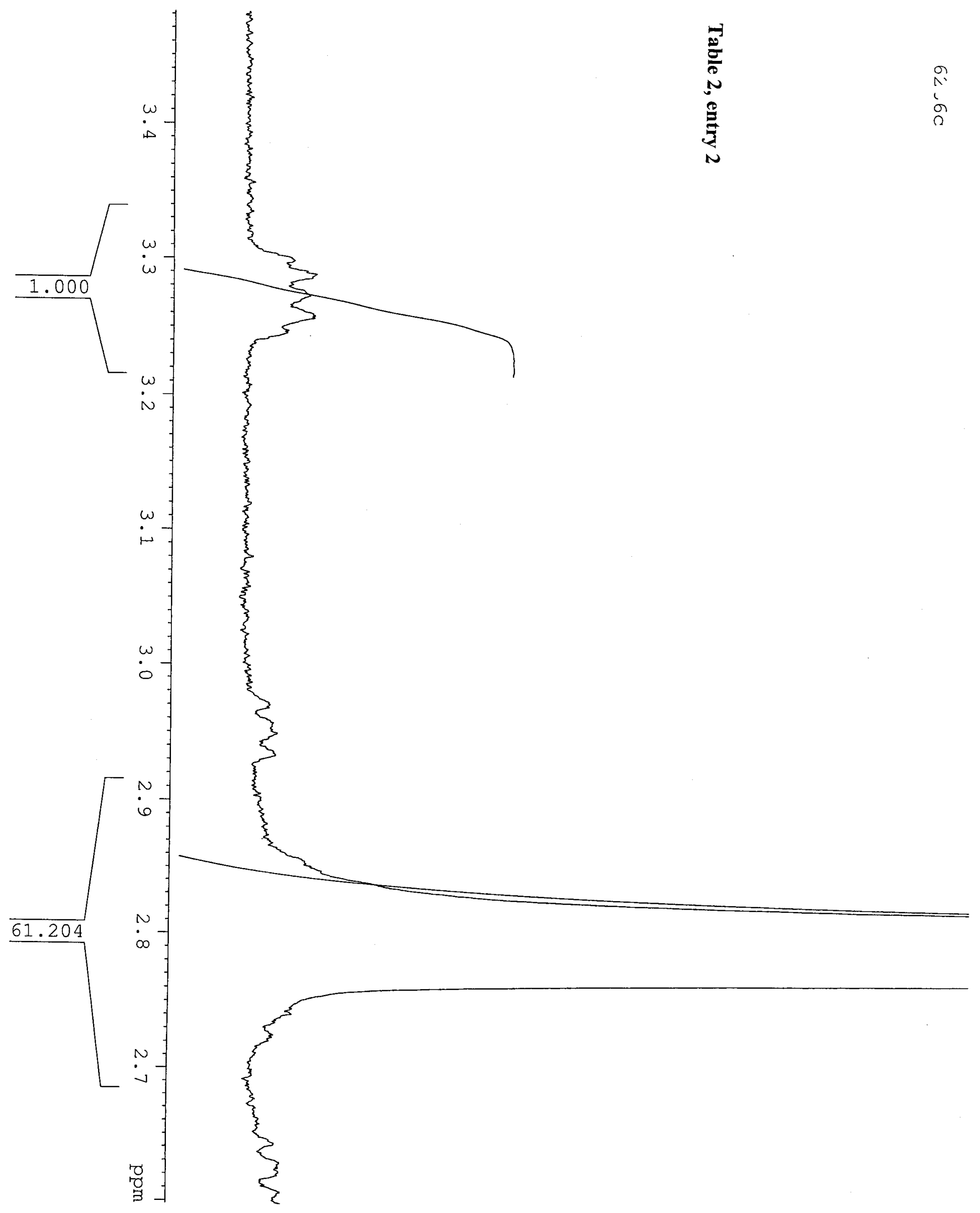




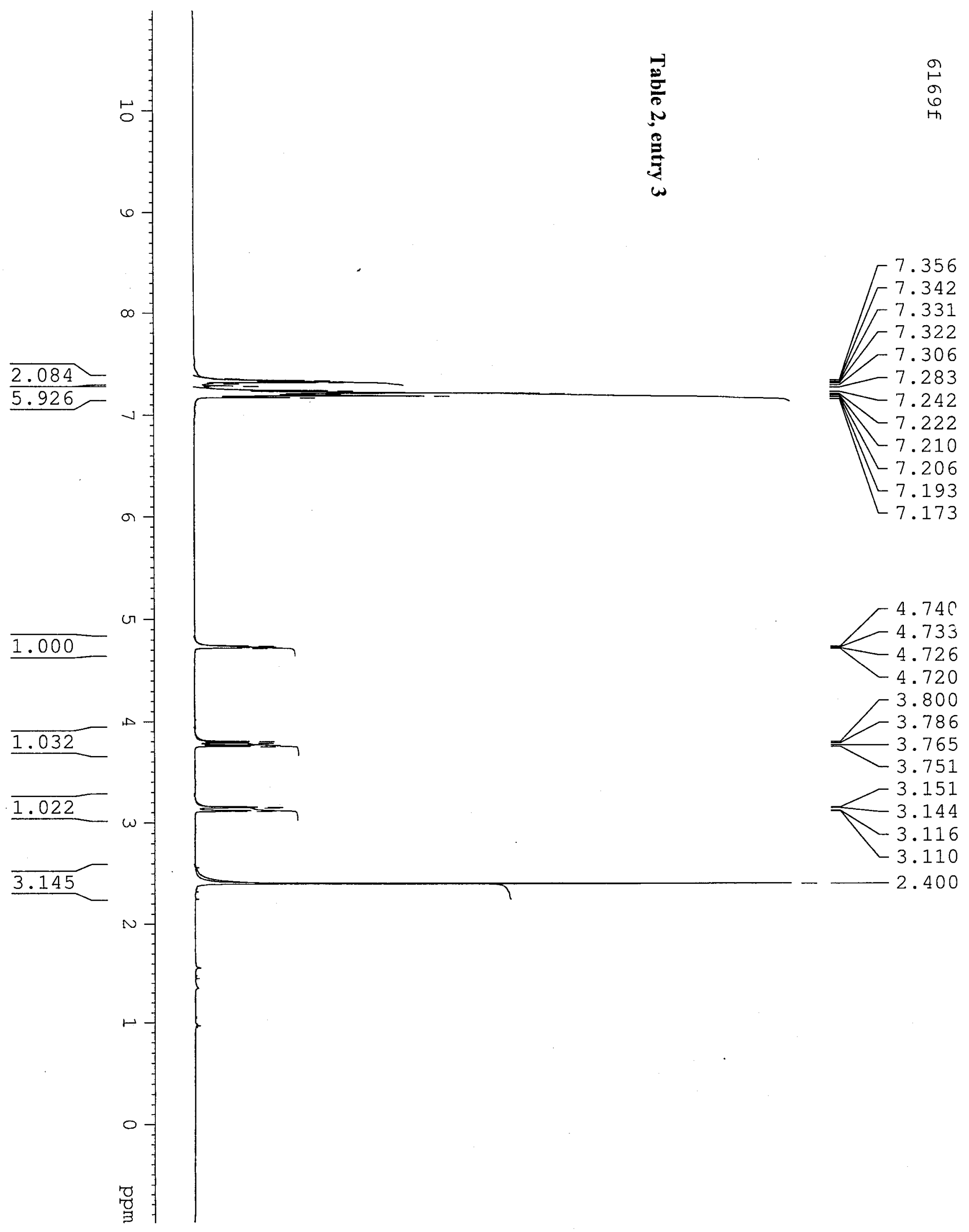




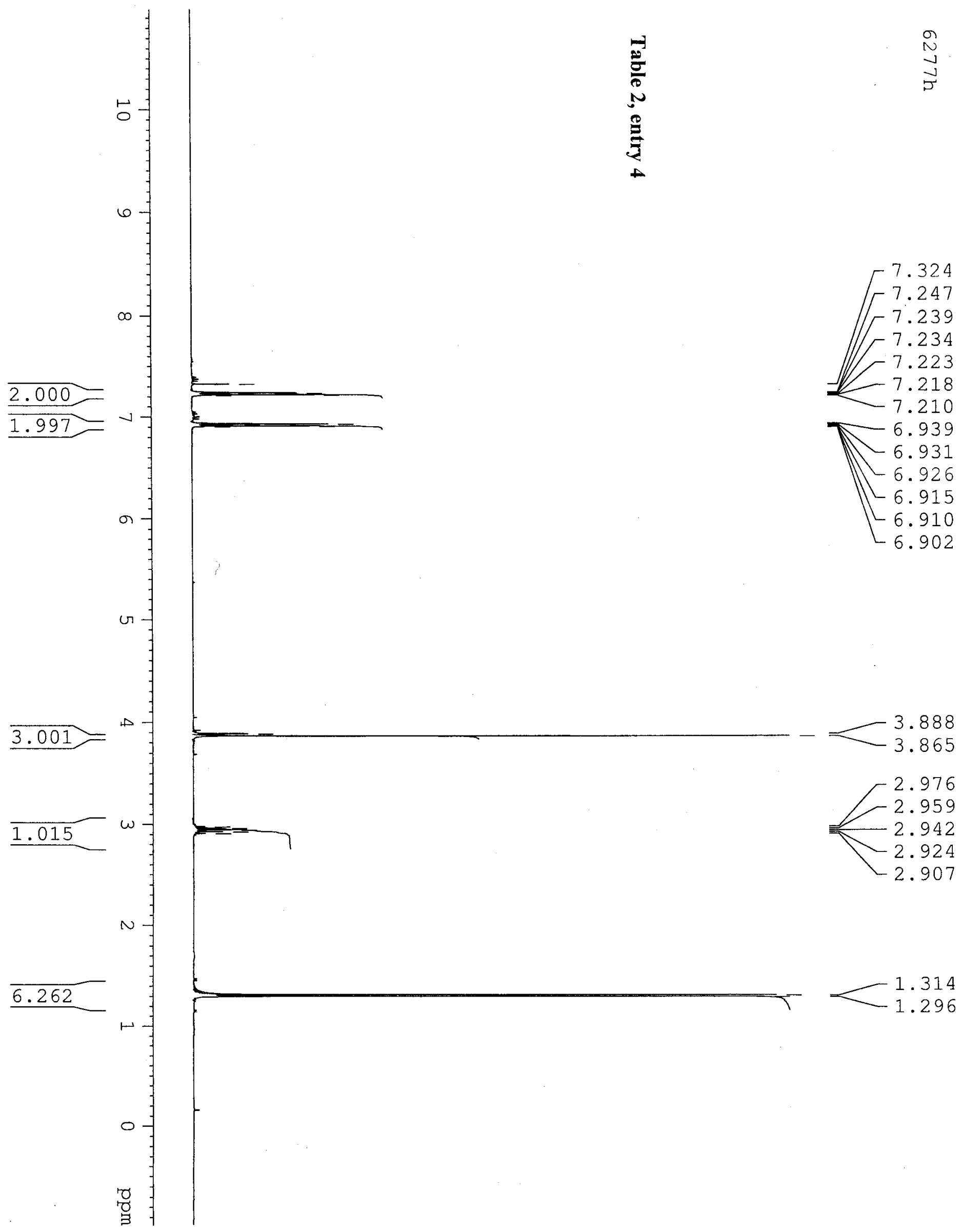




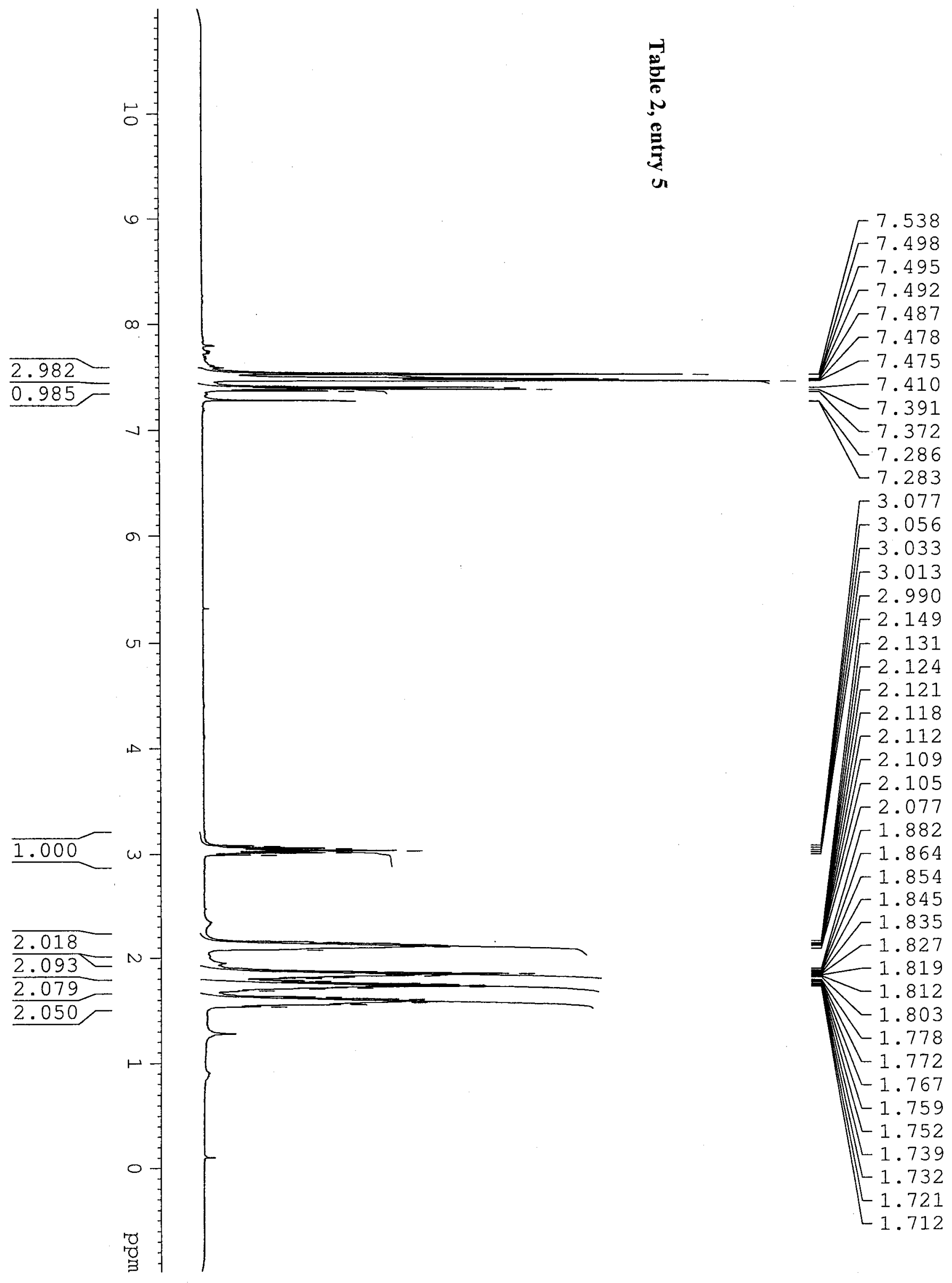




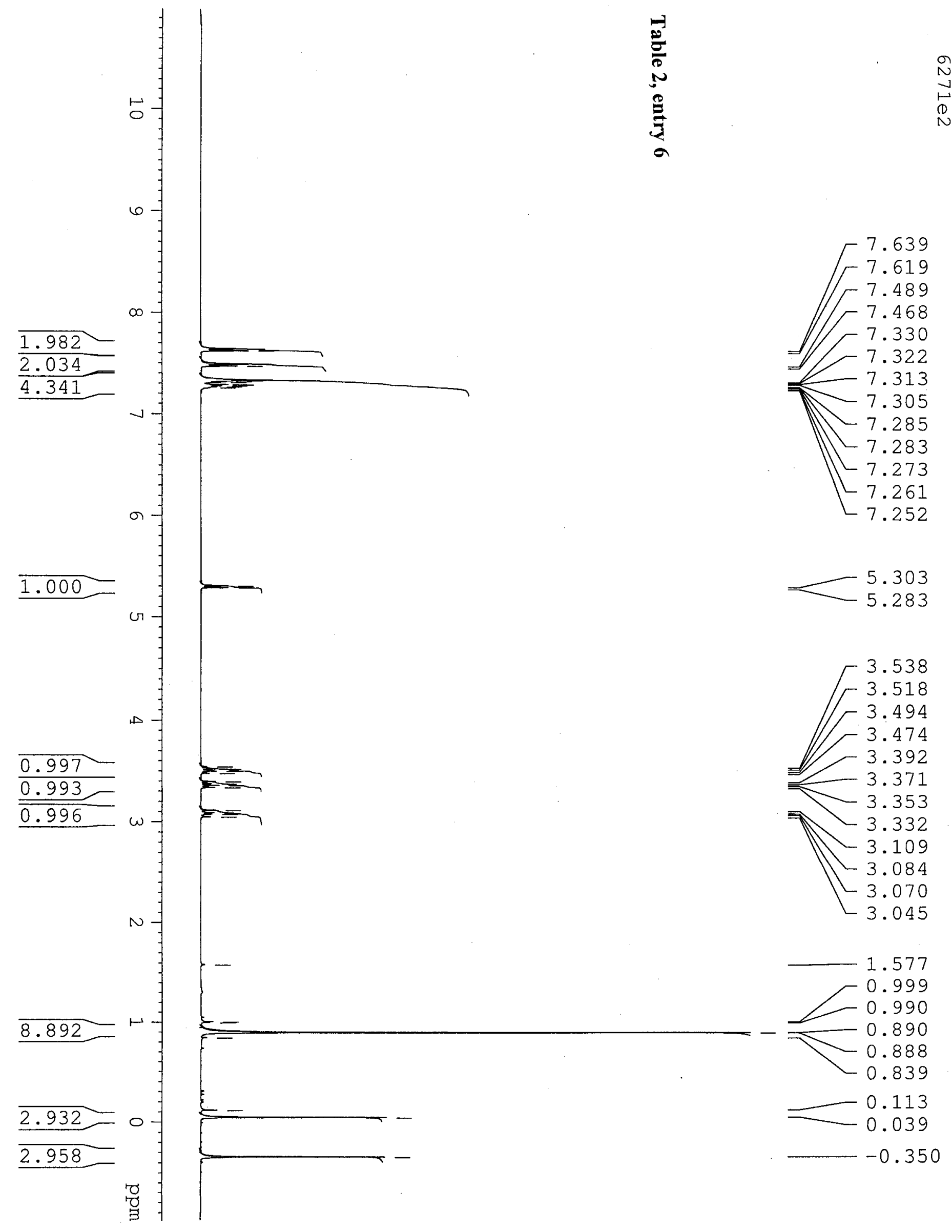




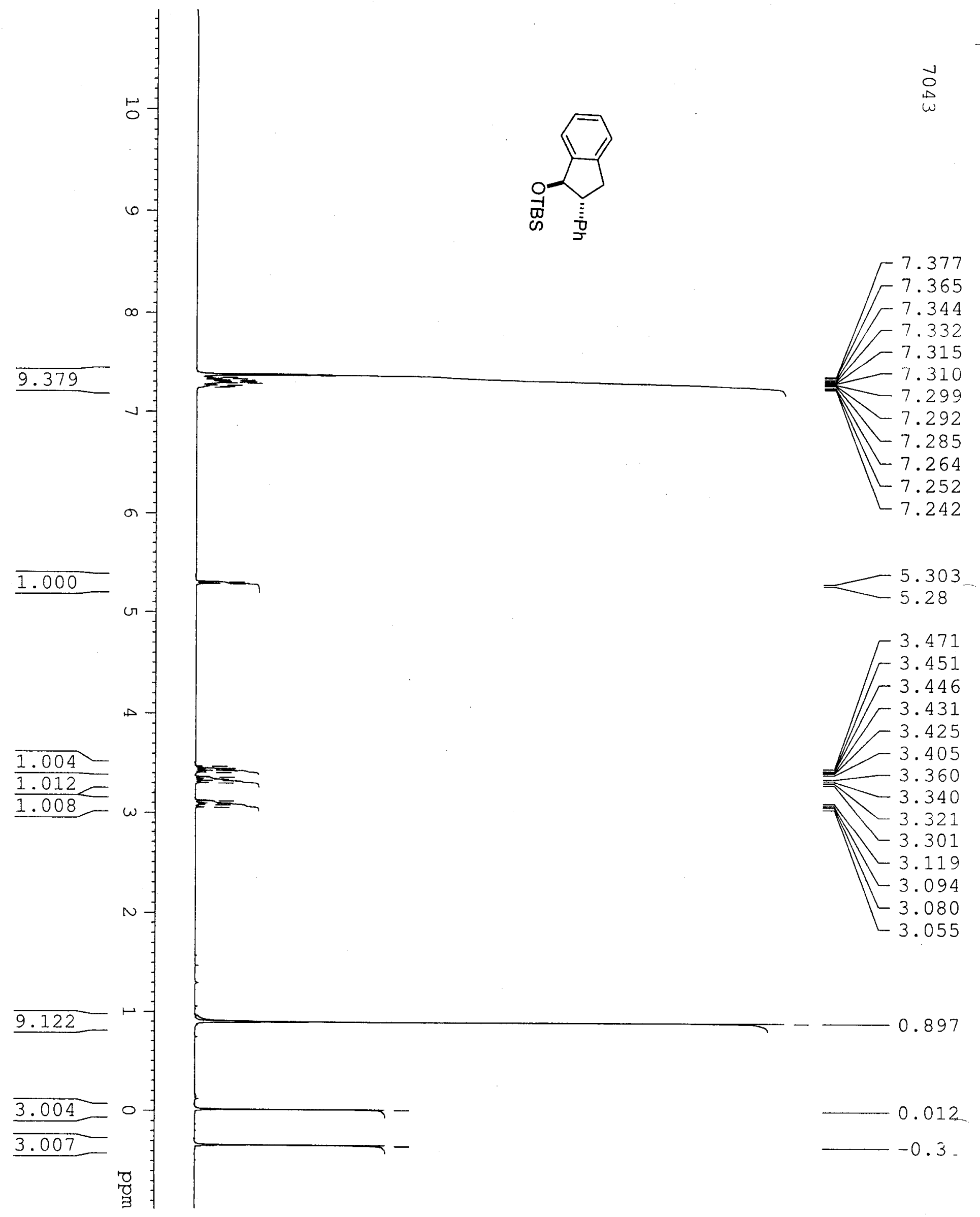




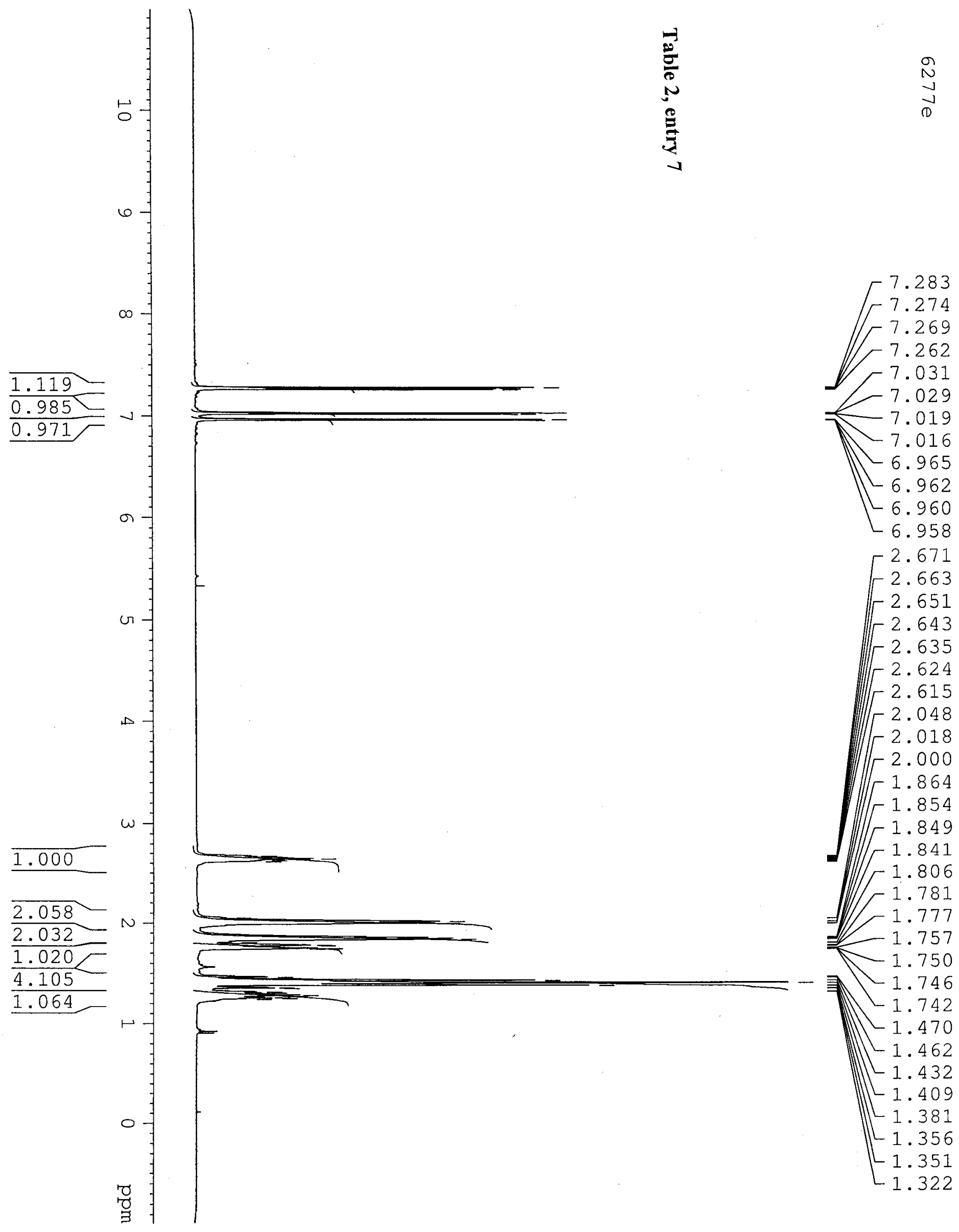




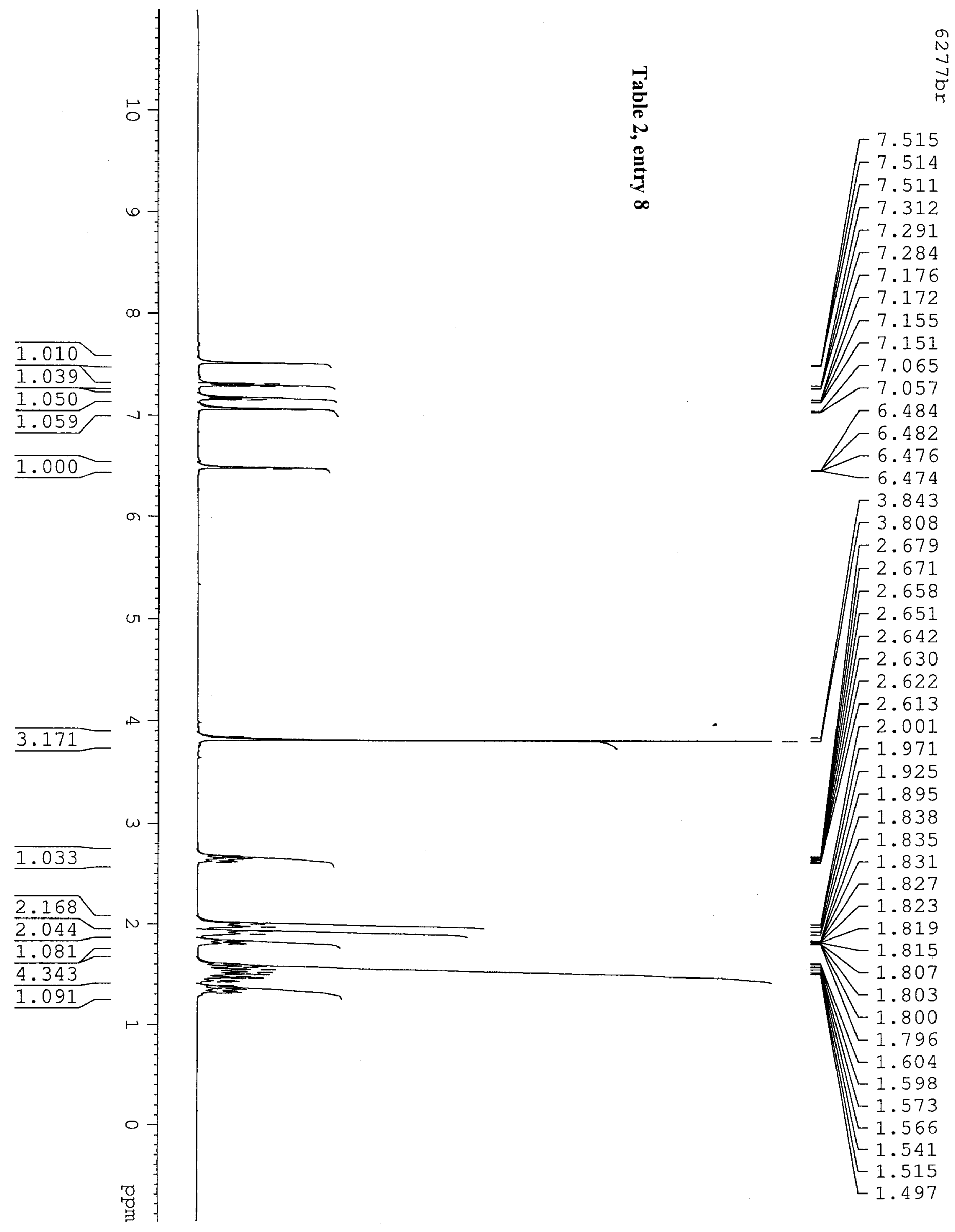




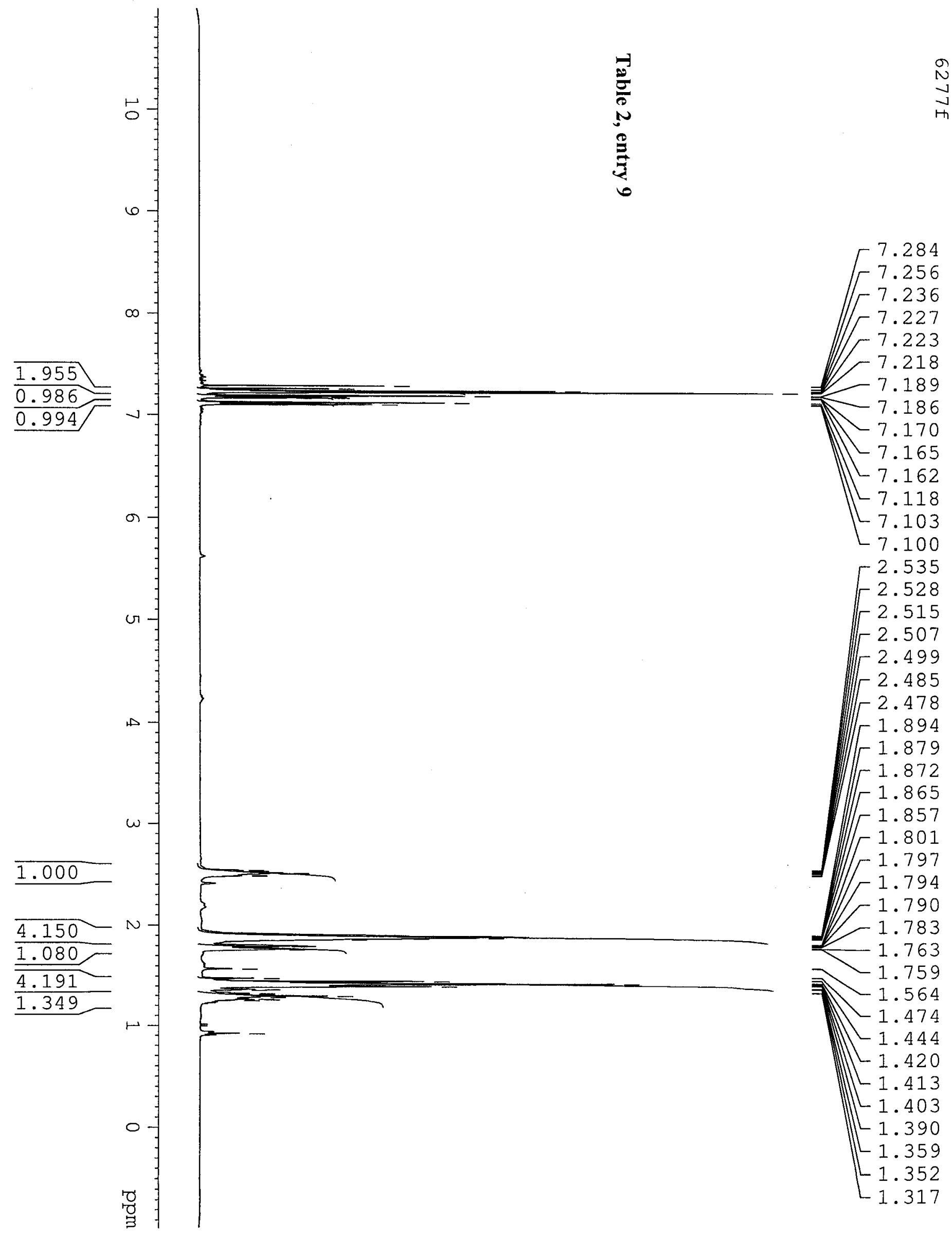




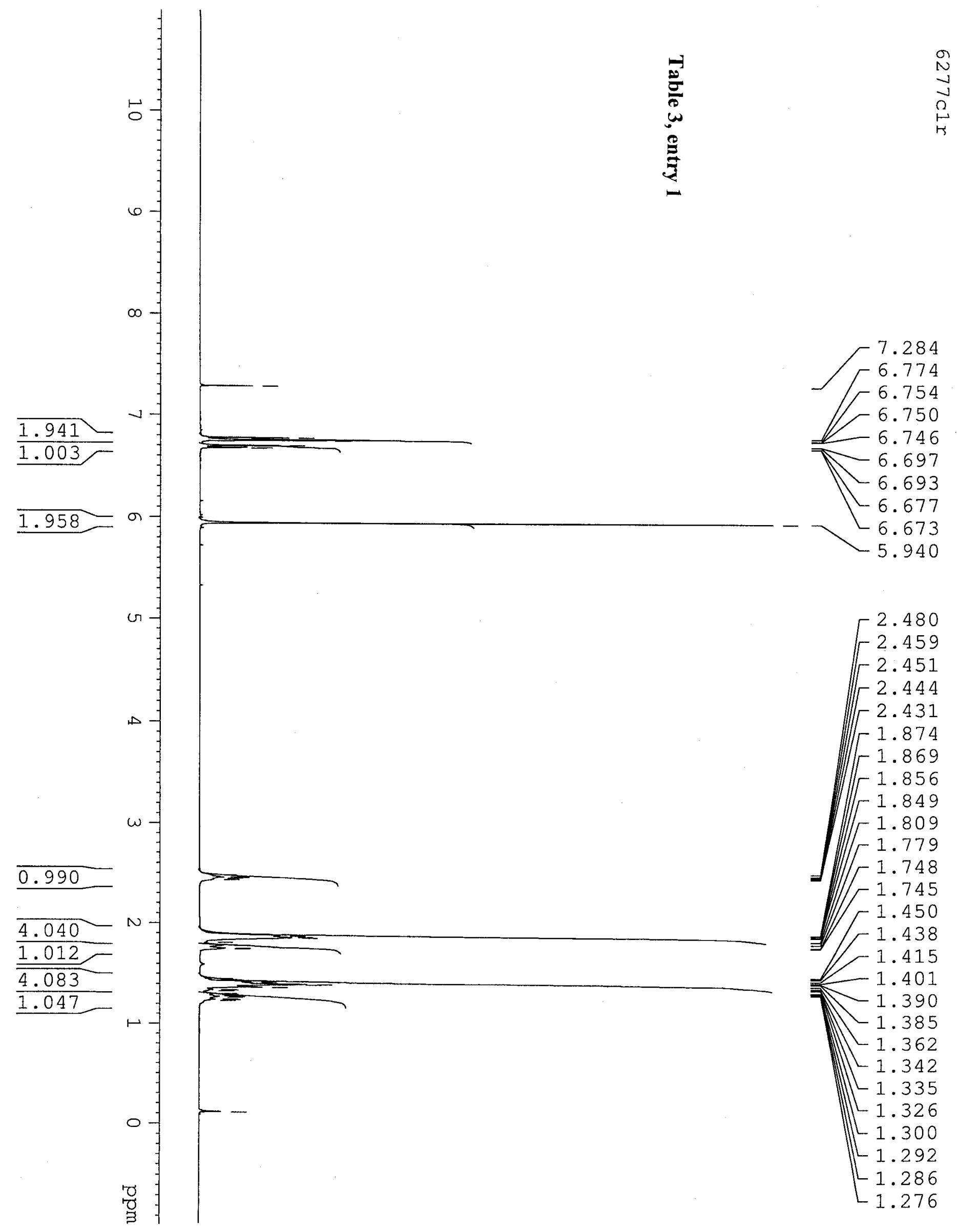




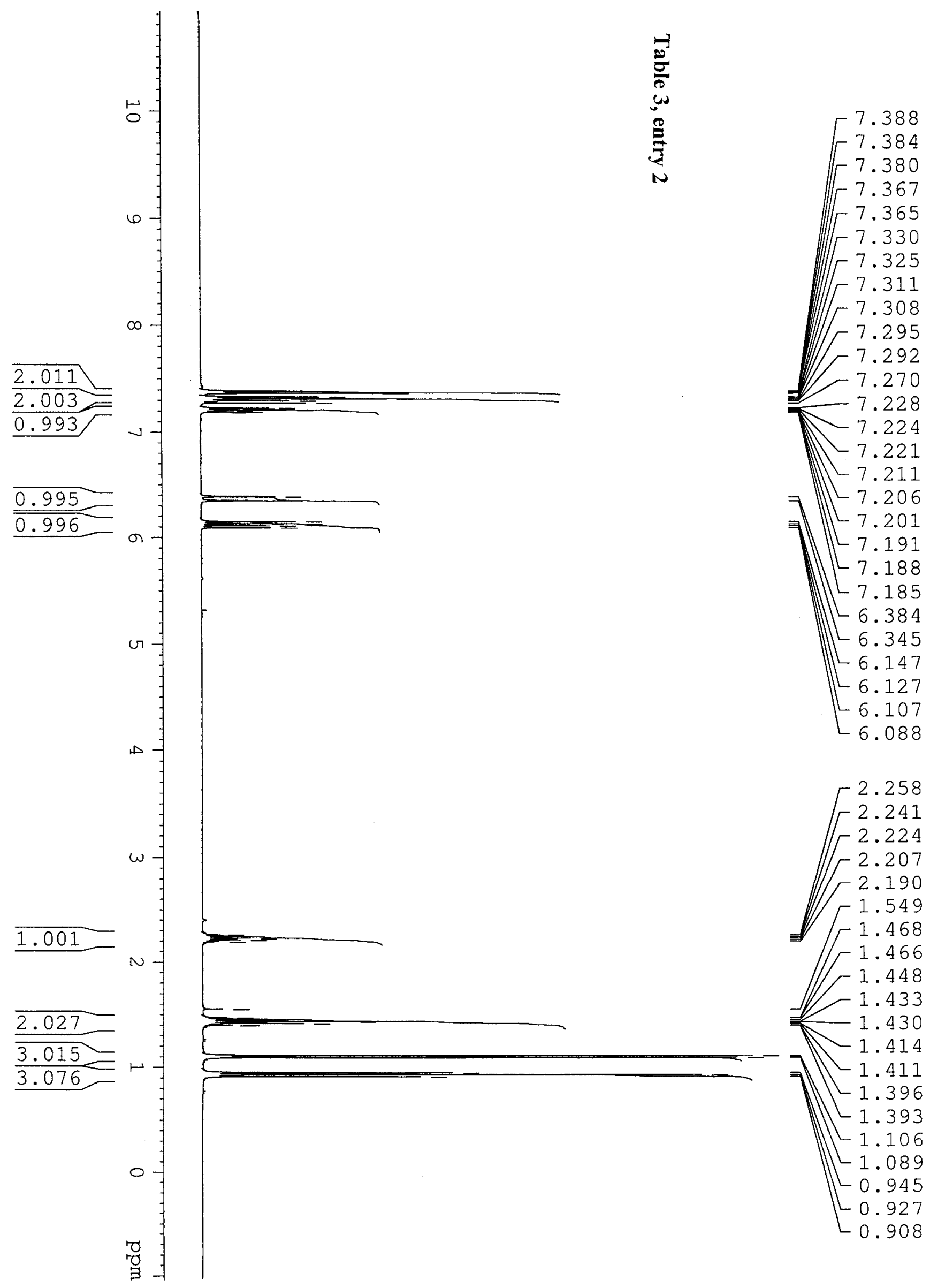




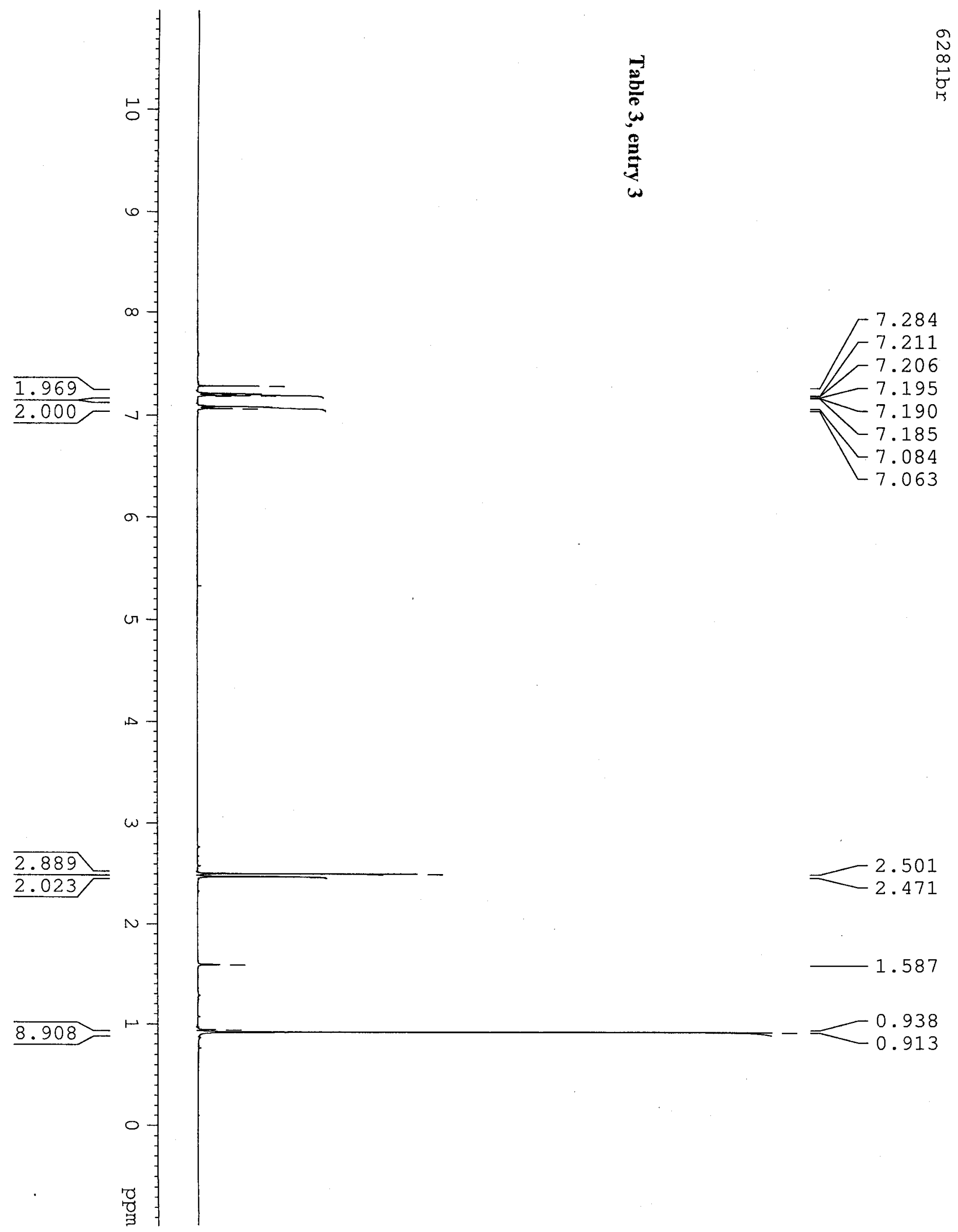




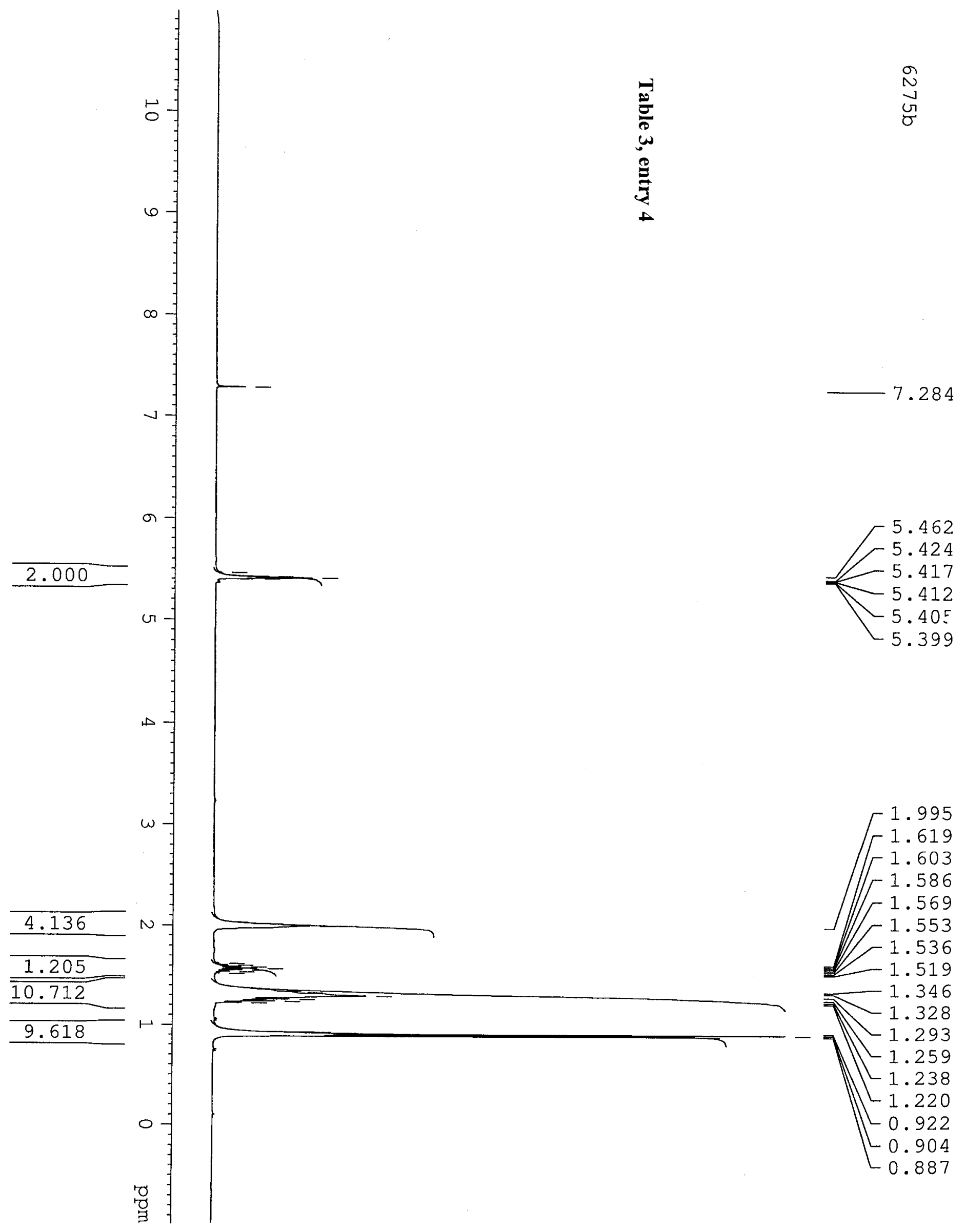

